\title{
The influence of Copernicus on physics
}

\section{B. Paczynski, Institute of Astronomy, Warsaw}

This month sees the 500th Anniversary of the birth of Copernicus. To mark the occasion, Europhysics News has invited B. Paczynski, Polish Board member of the EPS Division on Physics in Astronomy, to write this article.

Copernicus shifted the centre of the Universe from Earth to the Sun, and by doing so he deprived mankind of its unique position in the Universe. That action had the most profound impact on scientific thinking. It is likely that the philosophical consequences were even more important than the purely scientific value of the new model proposed for the solar system. The Copernican model helped people to look at the Universe from a new point of view : we may consider ourselves to be just a part of the Universe which can exist without us just as well, rather than to think that we are the very central and the most important part of the Universe. I think we should make a distinction between the scientific achievement and the philosophical consequences.

The Copernican model of the solar system, with some improvements due to Kepler, is still valid after four and a half centuries. There are not many scientific models created in this century which are likely to survive for so long. The heliocentric theory, once so radical, today belongs to the establishment. As time has passed by mankind has moved further and further away from any place that could be considered to be a centre of anything. It is surprising to notice how this extension of Copernican thinking has become a dogma of modern science.

Let us consider cosmology. Almost all cosmological theories adopt the so called cosmological principle: the Universe should look more or less the same everywhere. This is a simple extension of the idea that can be traced back to Copernicus. If Earth does not occupy a special position, then there is no reason for the Sun to be a unique star, and as a matter of fact the Galaxy we live in should not be a unique galaxy. The cosmological principle is basic for the theory, because it makes this theory sufficiently simple to study. It is a philosophical principle rather than a scien- tific one, and there are some perils associated with it.

Why should we not make the cosmological principle more perfect? Once we say that the Universe is the same everywhere, should we not also say that it is the same at all times? Once we have decided our place is not unique, why should the time we live in be unique? In fact such a strong cosmological principle has been adopted by some scientists. It leads directly to steady state cosmology. I believe this is the most extreme extension of the Copernican idea.

We live in the century when cosmology is becoming a science. Cosmological principles may be verified observationally. Observations of distant galaxies indicate that the Universe expands on a time scale of $10^{10}$ years. Therefore the density of matter decreases with time, contrary to the strong cosmological principle. To save this principles, we have to postulate a continuous creation of matter. This raises some philosophical problems. We either have to accept the hypothesis that matter is created now, or we have to agree that our times are unique and the Universe was entirely different some $10^{10}$ years ago. It is very fortunate that this is no longer a matter of philosophy only, but is a problem that may be the subject of observational studies. It is now possible to observe very faint

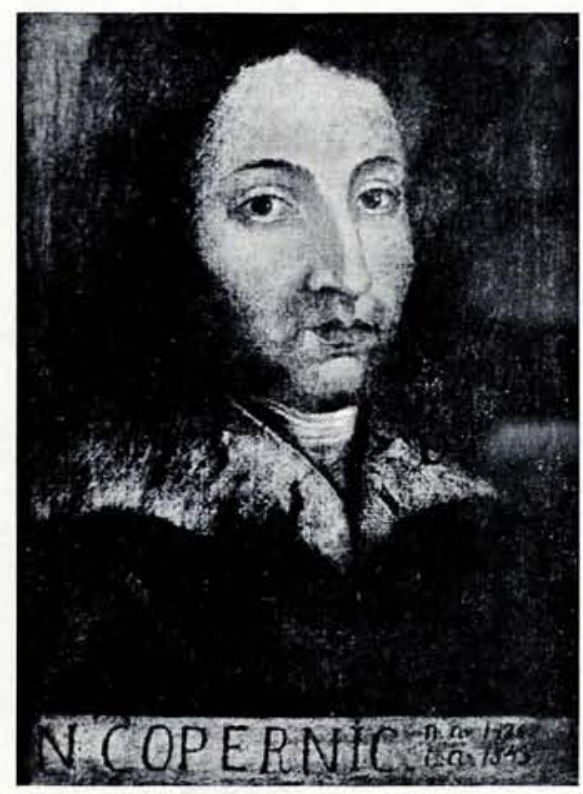

Nicolaus Copernicus 19 February 1473 - 24 May 1543 radiosources, objects believed to be extremely distant. Counts of these sources indicate that their number density is different at large distances from what it is here. Or we may put it differently: the number density of radiosources was different a long time ago from what it is now. This is in contradiction with the strong cosmological principle. Also, the Universe seems to be filled with a black body microwave radiation which has, at present, the temperature of $2.7 \mathrm{~K}$. It is almost certain that this radiation was produced about $10^{10}$ years ago when the Universe was very dense and hot, and matter was in thermal equilibrium with radiation. Such conditions are vastly different from the present situation and this is another observational evidence against the strong cosmological principle. We can no longer belive that the Universe was always more or less the same. But this conclusion is difficult to accept for many people. Therefore, a model of the oscillating Universe has been developed. Perhaps we live at some specific phase of the present cycle of the oscillating Universe, but there was, and there will be, an infinite number of cycles, and our cycle is not really unique in an way... Isn't this another extreme extension of the Copernican idea?

As there seems to be disagreement between the strong cosmological principle and the observations, we have to be more modest in extending the way of thinking initiated by Copernicus. Perhaps we should ask ourselves if it is really justified to assume that the Universe is more or less the same everywhere. Isotropy of the background $2.7 \mathrm{~K}$ radiation indicates that the Universe is homogeneous on a very large scale, over the distance of $10^{10}$ light years. But there is not too much observational evidence for the homogeneity on a scale of say $10^{9}$ light years. What should also be kept in mind is that we have no observational evidence for the existence of any extraterrestrial life, intelligent life in particular. The very important problem of whether we humans are alone in the Universe has not yet been solved by science. The answer one is willing to give to this problem depends mainly on one's philosophical convictions. I believe that most scientists are willing to think that mankind is not unique. Ultimately it is so because most of us cannot accept the possibility that the Earth could have a central position from any point of view. Copernicus was most efficient in moving us away from any kind of centre. 